\title{
The Association between Age, Sex, and Hospital-Acquired Infection Rates: Results from the 2009-2011 National Medicare Patient Safety Monitoring System
}

\author{
Sheila Eckenrode, RN; ${ }^{1}$ Anila Bakullari, BS; ${ }^{1}$ Mark L. Metersky, MD; ${ }^{1,2}$ Yun Wang, PhD; ${ }^{3}$ \\ Michelle M. Pandolfi, MSW, MBA; ${ }^{1}$ Deron Galusha, MS $;^{1,4}$ Lisa Jaser, Pharm; ${ }^{5}$ Noel Eldridge, MS $^{6}$
}

\begin{abstract}
овлестіVе. To define the relationships between age, sex and hospital-acquired infection (HAI) rates in a national cohort of hospitalized patients.

METHODS. Analysis of chart-abstracted Medicare Patient Safety Monitoring System data from randomly selected medical records of patients hospitalized between January 1,2009, and December 31, 2011, for acute cardiovascular disease, pneumonia, or major surgery associated with 1 of 6 HAIs. Patients were stratified into 6 groups. We then analyzed the association of age, sex, and 2 outcomes; the rate of occurrence of HAI for patients who were at risk and the rate of patients having at least 1 HAI.
\end{abstract}

RESULTS. Among 85,461 patients, all groups except younger female surgical patients had higher catheter-associated urinary tract infection (CAUTI) rates than male patients. After adjustment for comorbidities, there was no overall evidence of higher HAI rates among elderly patients. In patients with acute cardiovascular disease, women had higher rates of HAIs. Among patients with pneumonia, there was no significant difference in the rate of HAIs among most age and sex groups. Among surgical patients, all age and sex groups had a significantly higher adjusted rate of developing at least 1 HAI except females 65 years of age or older. Similar results were seen for the outcome of the occurrence rate of HAIs.

CONCLUSIONS. There was not an overall increased risk of HAIs among older patients hospitalized for acute cardiovascular disease, pneumonia, and major surgery after adjustment for comorbidities. The relationship between sex and the rate of HAIs varied depending upon the underlying acute reason for hospitalization.

Infect Control Hosp Epidemiol 2014;35(S3):S3-S9

Hospital-acquired infections (HAIs) are a major cause of morbidity and mortality in hospitalized patients. It is estimated that 1 in 20 inpatients has an infection related to hospital care at any given time. HAIs cost the US healthcare system approximately 30 billion dollars annually. ${ }^{1}$ Numerous studies have attempted to define risk factors for HAIs. The frequency with which invasive devices are used and the length of time they remain in place directly influence the rate of many types of HAIs, including catheter-associated urinary tract infection (CAUTI), ${ }^{2}$ central line-associated bloodstream infection (CLABSI), ${ }^{3}$ and ventilator-associated pneumonia (VAP). ${ }^{4}$ Additionally, suboptimum practices related to isolation, ${ }^{5}$ procedural technique, ${ }^{5,6}$ and care of indwelling devices $^{3,6}$ contribute to the prevalence of certain HAIs.

Patient characteristics are also thought to influence the risk of development of various HAIs. Elderly individuals are at higher risk of developing HAIs, possibly in part because of immunosenescence, the age-related decline of the immune system. ${ }^{7}$ The higher prevalence of comorbidities in the elderly population likely plays an important role in the development of HAIs. Sex also plays a role in the development of certain HAIs; there is strong evidence of an increased risk of CAUTI in females, attributable to anatomic differences that result in a greater propensity for bacterial contamination of the catheter. $^{2}$ For HAIs other than CAUTI, the evidence is limited and sometime conflicting. For example, some studies have reported a higher risk for Clostridium difficile infection in males, ${ }^{8}$ whereas others have not. ${ }^{9}$ In a retrospective cohort study, Cohen et $\mathrm{al}^{10}$ found that men were at higher risk for bloodstream and surgical site infections, possibly because of

Affiliations: 1. Qualidigm, Wethersfield, Connecticut; 2. Division of Pulmonary and Critical Care Medicine, University of Connecticut School of Medicine, Farmington, Connecticut; 3. Department of Biostatistics, Harvard School of Public Health, Boston, Massachusetts; 4. Section of General Internal Medicine, Department of Internal Medicine, Yale University School of Medicine, New Haven, Connecticut; 5. Pharmacy, Griffin Hospital, Derby, Connecticut;

6. Agency for Healthcare Research and Quality, US Department of Health and Human Services, Rockville, Maryland.

Received April 23, 2014; accepted May 25, 2014; electronically published September 15, 2014.

(c) 2014 by The Society for Healthcare Epidemiology of America. All rights reserved. 0899-823X/2014/35S3-0002\$15.00. DOI: 10.1086/677831 
TABLE 1. Patient Characteristics

\begin{tabular}{|c|c|c|c|c|c|c|c|}
\hline \multirow[b]{2}{*}{ Characteristic } & \multirow[b]{2}{*}{$\begin{array}{c}\text { Aggregate } \\
(n=85,461)\end{array}$} & \multicolumn{3}{|c|}{ Female sex } & \multicolumn{3}{|c|}{ Male sex } \\
\hline & & $\begin{array}{l}\text { Age } 18-44 \text { years } \\
(n=5,218)\end{array}$ & $\begin{array}{c}\text { Age } 45-64 \text { years } \\
(n=12,988)\end{array}$ & $\begin{array}{c}\text { Age } \geq 65 \text { years } \\
(n=28,189)\end{array}$ & $\begin{array}{l}\text { Age } 18-44 \text { years } \\
(n=2,789)\end{array}$ & $\begin{array}{c}\text { Age } 45-64 \text { years } \\
(n=12,896)\end{array}$ & $\begin{array}{c}\text { Age } \geq 65 \text { years } \\
(n=23,381)\end{array}$ \\
\hline Percentage of aggregate patients & 100.0 & 6.1 & 15.2 & 33.0 & 3.3 & 15.1 & 27.4 \\
\hline \multicolumn{8}{|l|}{ Condition } \\
\hline \multicolumn{8}{|l|}{ Acute cardiovascular disease } \\
\hline Proportion & $35,270 / 85,461$ & $507 / 35,270$ & $3,521 / 35,270$ & $12,908 / 35,270$ & $985 / 35,270$ & $6,090 / 35,270$ & $11,259 / 35,270$ \\
\hline Percentage & 41.3 & 1 & 10 & 37 & 3 & 17 & 32 \\
\hline \multicolumn{8}{|l|}{ Pneumonia } \\
\hline Proportion & $25,034 / 85,461$ & $1,229 / 25,034$ & $3,403 / 25,034$ & $8,486 / 25,034$ & $1,122 / 25,034$ & $3,176 / 25,034$ & $7,618 / 25,034$ \\
\hline Percentage & 29.3 & 5 & 14 & 34 & 4 & 13 & 30 \\
\hline \multicolumn{8}{|l|}{ Major surgery } \\
\hline Proportion & $25,157 / 85,461$ & $3,482 / 25,157$ & $6,064 / 25,157$ & $6,795 / 25,157$ & $682 / 25,157$ & $3,630 / 25,157$ & $4,504 / 25,157$ \\
\hline Percentage & 29.4 & 14 & 24 & 27 & 3 & 14 & 18 \\
\hline \multicolumn{8}{|l|}{ Comorbidity, $\%$ of patients } \\
\hline Cancer & 19.3 & 6.2 & 14.8 & 21.2 & 5.7 & 13.4 & 27.3 \\
\hline Diabetes & 35.2 & 12.7 & 34.4 & 36.7 & 19.5 & 36.2 & 40.2 \\
\hline Obesity & 23.3 & 27.0 & 35.4 & 19.7 & 27.9 & 28.1 & 17.0 \\
\hline Cerebrovascular disease & 16.2 & 2.3 & 8.8 & 21.6 & 3.5 & 9.9 & 21.9 \\
\hline CHF/pulmonary edema & 40.9 & 8.5 & 24.4 & 51.9 & 21.3 & 30.9 & 51.7 \\
\hline COPD & 30.6 & 7.1 & 26.2 & 33.7 & 10.3 & 26.2 & 39.6 \\
\hline Smoking & 23.8 & 36.9 & 33.5 & 11.6 & 47.6 & 27.7 & 16.5 \\
\hline Renal disease & 25.7 & 5.4 & 14.2 & 29.8 & 14.1 & 19.7 & 36.6 \\
\hline
\end{tabular}

NOTE. Data are proportion (\%) of patients, unless otherwise indicated. CHF, congestive heart failure; COPD, chronic obstructive pulmonary disease.

differences in propensity for skin colonization or anatomical differences. Other studies have not detected a higher rate of CLABSI in males. ${ }^{11}$

The Medicare Patient Safety Monitoring System (MPSMS) includes information on 21 patient safety measures abstracted from a randomly selected all-payer national sample of charts of patients hospitalized in acute care hospitals from 2002 to 2012 , with the exception of 2008 , when no data were abstracted. ${ }^{12,13}$ These measures include $6 \mathrm{HAI}$ indicators reported in the current study; CLABSI, CAUTI, hospitalacquired C. difficile, sterile site methicillin-resistant Staphylococcus aureus (MRSA), postoperative pneumonia, and VAP. Enhanced understanding of the risk factors and epidemiology of HAIs is an important basis for preventative efforts. This study was designed to explore sex- and agerelated differences in specific HAI rates among a large, randomly selected national cohort of patients while controlling for patient characteristics and common comorbidities.

\section{METHODS}

\section{Study Sample}

Our study sample was drawn from the 2009-2011 MPSMS data, which included patients 18 years of age and older discharged from an acute care hospital for 4 conditions: acute myocardial infarction (AMI), heart failure (HF), pneumonia, and conditions requiring major surgery as defined by the Surgical Care Improvement Project. For this study, we com- bined patients with AMI and patients with HF together as "acute cardiovascular disease."

\section{Patient Characteristics}

Patient characteristics include demographic characteristics of age, sex, and race as well as clinical comorbidities. Age was categorized into 3 groups: (1) 18-44 years, (2) 45-64 years, and (3) 65 years or older. Sex was coded as female or male. Clinical comorbidities were identified from the medical records and include heart failure, obesity, coronary artery disease, renal disease, cerebrovascular disease, chronic obstructive pulmonary disease, cancer, diabetes, and smoking.

\section{HAI Measures and Outcomes}

The 6 HAI measures are (1) CLABSI, (2) postoperative pneumonia, (3) CAUTI, (4) sterile site hospital-acquired MRSA, (5) hospital-acquired C. difficile, and (6) VAP. Definitions and algorithms to identify these HAIs differ from those used in the Centers for Diseases Control and Prevention National Healthcare Safety Network (NHSN) and are available online (http://www.qualidigm.org/index.php/current-initiatives/ mpsms) and in the on-line appendix to a recent publication. ${ }^{13}$ Our primary interests were 2 composite outcomes of these 6 HAIs: the rate of occurrence of adverse events for which patients were at risk, and the rate of patients with 1 or more adverse events, as described in a previous study. ${ }^{13}$ Not all patients were at risk for all HAIs. For example, only patients who underwent bladder catheterization were at risk for 
TABLE 2. Rates of Catheter-Associated Urinary Tract Infection (CAUTI)

\begin{tabular}{lccc}
\hline & \multicolumn{2}{c}{ No. of patients with CAUTIs/no. of patients at risk (\% [95\% CI]) } \\
\cline { 2 - 4 } Variable & Age $18-44$ years & Age $45-64$ years & Age $\geq 65$ years \\
\hline $\begin{array}{l}\text { Pneumonia } \\
\quad \text { Male }\end{array}$ & $5 / 156(3.2[1.2-7.5])$ & $11 / 723(1.5[0.8-2.7])$ & $81 / 2,113(3.8[3.1-4.7])$ \\
$\quad \begin{array}{l}\text { Female } \\
\text { Acute cardiovascular disease }\end{array}$ & $7 / 142(4.9[2.2-10.0])$ & $29 / 778(3.7[2.6-5.3])$ & $115 / 2,663(4.3[3.6-5.2])$ \\
$\quad$ Male & $3 / 138(2.2[0.5-6.5])$ & $33 / 1,208(2.7[1.9-3.8])$ & $142 / 3,347(4.2[3.6-5.0])$ \\
$\quad$ Female & $9 / 121(7.4[3.8-13.7])$ & $55 / 1,099(5.0[3.9-6.5])$ & $355 / 5,315(6.7[6.0-7.4])$ \\
Major surgery & & & \\
$\quad$ Male & & & \\
$\quad$ Female & $8 / 577(1.3[0.7-2.8])$ & $29 / 3,011(1.0[0.7-1.4])$ & $103 / 3,809(2.7[2.2-3.3])$ \\
\end{tabular}

NOTE. CI, confidence interval.

CAUTI, and only patients with mechanical ventilation were at risk for VAP.

\section{Statistical Analysis}

We fitted a mixed-effects model with a logit link function to assess the relationship of age-sex and the rate of occurrence of adverse events and the rate of patients with 1 or more adverse events. Models were fitted with hospital-specific random intercepts to account for within-hospital and betweenhospital variations and adjusted for patient characteristics (age, race, and comorbidities). All models included 5 dummy variables: male and age 18-44 years, male and age 45-64 years, female and age 18-44 years, female and age 45-64 years, female and aged 65 years and older, with the reference group being males aged 65 years and older. Models were fitted for patients with cardiovascular disease, pneumonia, and major surgery separately. Analyses were conducted using SAS version 9.2 (SAS Institute). We obtained institutional review board approval through Solutions IRB (http://www solutionsirb.com), which waived the requirement for informed consent.

\section{RESULTS}

The final study sample included 85,461 individual patients, 35,270 with AMI/HF, 25,034 with pneumonia, and 25,157 surgical patients. Table 1 demonstrates the demographic characteristics and comorbidities of the study patients. Patients with acute cardiovascular disease comprised $41.3 \%$ of the sample, patients with pneumonia comprised $29.3 \%$, and patients with major surgery comprised $29.4 \%$. Heart failure and/ or pulmonary edema was the most frequently occurring comorbidity (40.9\%), followed by diabetes (35.2\%) and chronic obstructive pulmonary disease $(30.6 \%)$ for the total sample. Male and female patients in the 65 years of age or older group had higher rates of all comorbidities except smoking and obesity.

The unadjusted rates of HAIs for which the patients were at risk are presented in Tables 2 and 3. CAUTIs represent $1,294(52.8 \%)$ of all 2,449 HAIs detected. We present the CAUTI rates in Table 2 and a composite rate of the 5 other HAIs in Table 3. For all age groups and underlying conditions, except surgical patients 18-44 years of age, females were at higher risk of CAUTI than males. The relationship between

TABLE 3. Composite Rates of Other Hospital-Acquired Infections (HAIs)

\begin{tabular}{|c|c|c|c|}
\hline \multirow[b]{2}{*}{ Variable } & \multicolumn{3}{|c|}{ No. of patients with infection/no. of patients at risk (\% $[95 \% \mathrm{CI}])$} \\
\hline & Age $18-44$ years & Age $45-64$ years & Age $\geq 65$ years \\
\hline \multicolumn{4}{|c|}{ Pneumonia } \\
\hline Male & $11 / 2,225(0.5[0.3-0.9])$ & $39 / 6,294(0.6[0.5-0.9])$ & $98 / 14,826(0.7[0.4-1.3])$ \\
\hline Female & $0 / 2,431(0.0[0.0-0.2])$ & $36 / 6,771(0.5[0.4-0.7])$ & $92 / 16,566(0.6[0.2-1.1])$ \\
\hline \multicolumn{4}{|c|}{ Acute cardiovascular disease } \\
\hline Male & $6 / 1,361(0.4[0.2-1.0])$ & $48 / 9,215(0.5[0.4-0.7])$ & $101 / 17,632(0.6[0.3-1.1])$ \\
\hline Female & $1 / 750(0.1[0.0-0.8])$ & $25 / 5,400(0.5[0.3-0.7])$ & $58 / 20,234(0.3[0.1-0.7])$ \\
\hline \multicolumn{4}{|c|}{ Major surgery } \\
\hline Male & $26 / 2,149(1.2[0.8-1.8])$ & $99 / 11,451(0.8[0.4-1.6])$ & $205 / 14,364(1.4[1.0-2.2])$ \\
\hline Female & $26 / 10,388(0.2[0.1-0.8])$ & $81 / 18,493(0.4[0.2-0.9])$ & $216 / 21,114(1.0[0.7-1.6])$ \\
\hline
\end{tabular}




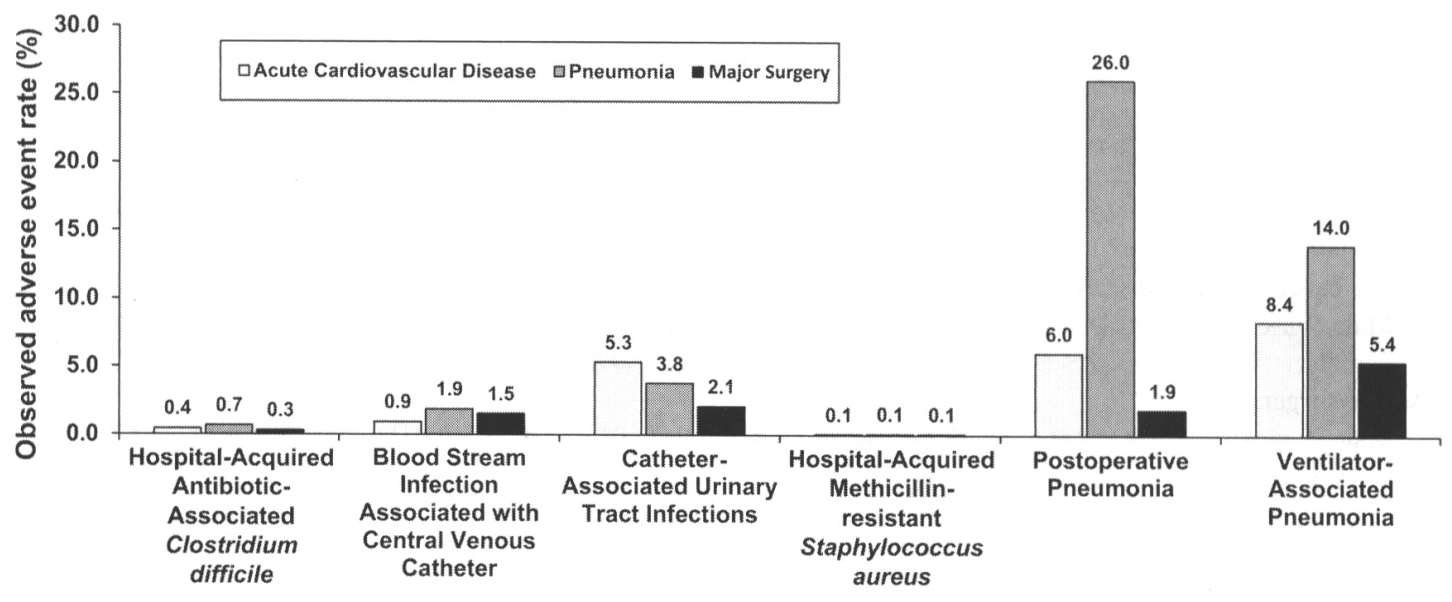

FIGURE 1. Observed adverse event rate, age 18 years and older, both sexes, by condition.

age and CAUTI rates varied between patient groups and sex. In Table 3, representing a composite rate for CLABSI, postoperative pneumonia, MRSA, $C$. difficile and VAP, it can be seen that, for most groups, females have lower rates of HAIs. The association between age and HAI rates is less consistent. The unadjusted rates demonstrate higher HAI rates in older surgical patients but no obvious age-related trend for the other 2 conditions.

Figure 1 represents the observed adverse event rate (\%) for all included ages and both sexes by condition. Figure $2 \mathrm{~A}$ illustrates the adjusted odds ratios of occurrence of any HAI by age and sex group. Compared with the reference group of men aged 65 years and older, the adjusted rate of occurrence of HAIs varied depending on the underlying acute clinical condition. Among patients with acute cardiovascular disease, women of any age had a higher adjusted HAI rate. However, among patients with pneumonia, younger women had a lower rate of HAIs, and all other groups had similar HAI rates. Among surgical patients, all groups had similar adjusted HAIs rates, except for younger males, who had a markedly higher adjusted rate of HAIs. Overall, the riskadjusted rates do not demonstrate higher rates of HAIs in the older patients. Figure $2 B$ illustrates the odds ratio of occurrence of at least 1 HAI by age and sex group. When compared with Figure $2 A$, similar patterns are seen, with the exception of younger females in the surgical group having significantly higher rates of HAI.

\section{DISCUSSION}

In our large, nationally representative study of age-sex disparities in HAIs, we found variation in the rate of HAIs dependent on the specific HAI (CAUTI vs a composite rate of other HAIs). The association between age and sex and adjusted HAI rates was complex but demonstrated no clear age-dependent trend. Furthermore, the age and sex-related variation in HAI rates was dependent upon the patient's underlying acute medical condition. In patients with acute car- diovascular disease, women had higher rates of HAIs. Among patients with pneumonia, young women had the lowest risk, whereas men and women of other age groups had similar rates. Among surgical patients, young men had the highest rate of HAIs. Young women who were surgical patients may include a large number of healthy women who received hysterectomies and may be at lower risk of HAIs than men in the same age group who are surgical patients. Overall, older patients did not have higher risk-adjusted HAI rates. These results diverge from several earlier studies in which HAI rates were the highest among elderly patients, ${ }^{2,7-9}$ although other studies have not detected a relationship between age and some HAIs. ${ }^{4,14}$ One potential explanation for not finding an increased rate of HAIs among the elderly population is that we only included in our denominator those patients who were at risk for a specific infection, unlike studies that used all hospitalized patients as their denominator. ${ }^{7}$ For example, if elderly patients are at increased risk of CLABSI only because of an increased central venous catheterization rate relative to younger patients, our analyses would not detect that. Similarly, patients known to be colonized with MRSA at the time of admission were excluded from the MRSA denominator in our study. However, it seems most appropriate to explore HAI rates only among the patient population at risk for the HAI.

Although this study cannot determine the underlying reasons for the variation in HAI rates observed, several factors likely contribute. Like others, ${ }^{2}$ we found a higher unadjusted rate of CAUTI in females than in males. This may be driving our observation of a higher adjusted rate of HAIs among females (compared with males) with cardiovascular disease and surgical conditions (except in females 65 years of age and older). This relationship was not seen in females with pneumonia, possibly related to the fact that patients with pneumonia receive systemic antibiotic treatment, which may prevent certain HAIs, including VAP ${ }^{15}$ and CAUTI. ${ }^{16}$ Although there are not likely to be sex-specific differences in 


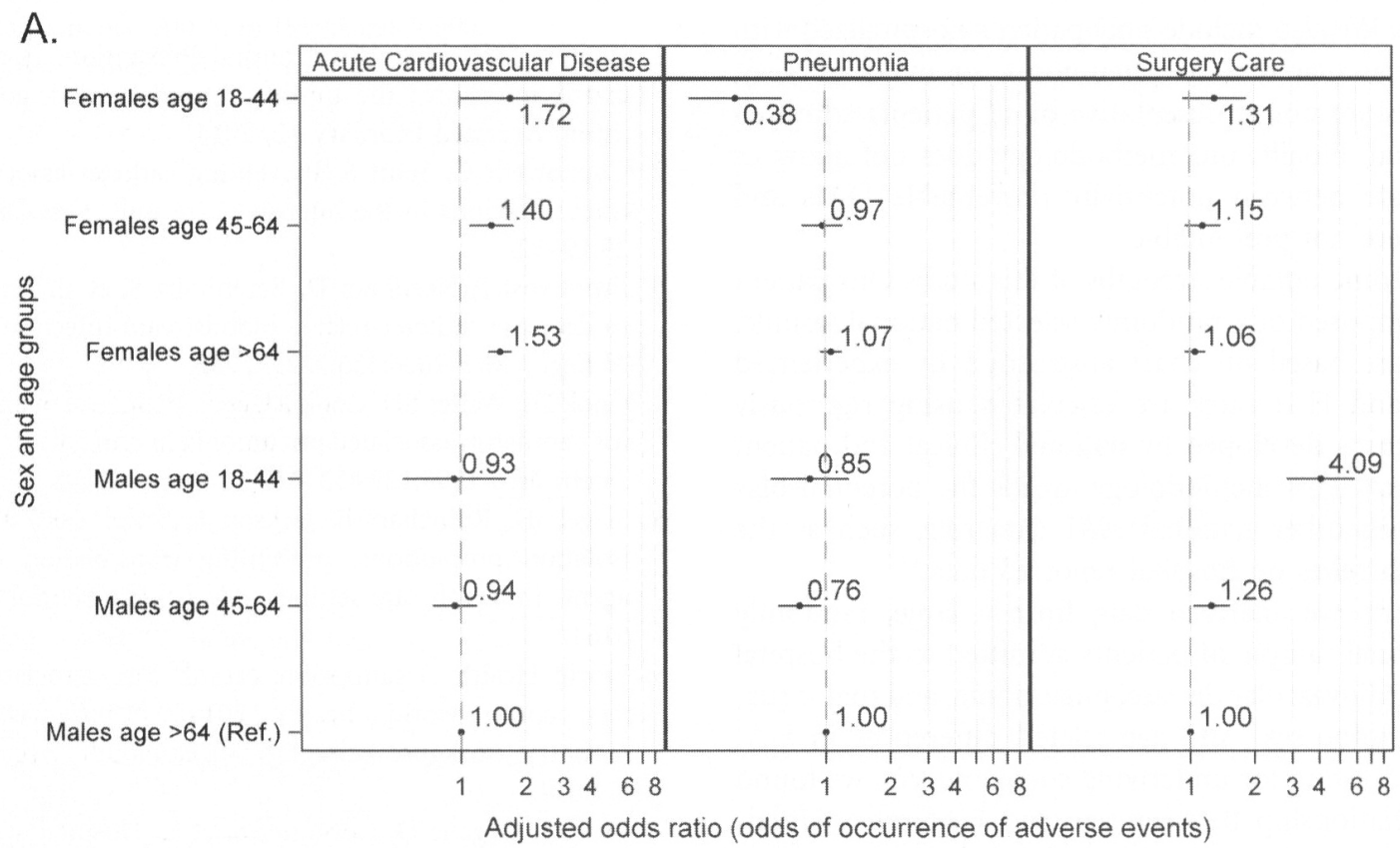

B.

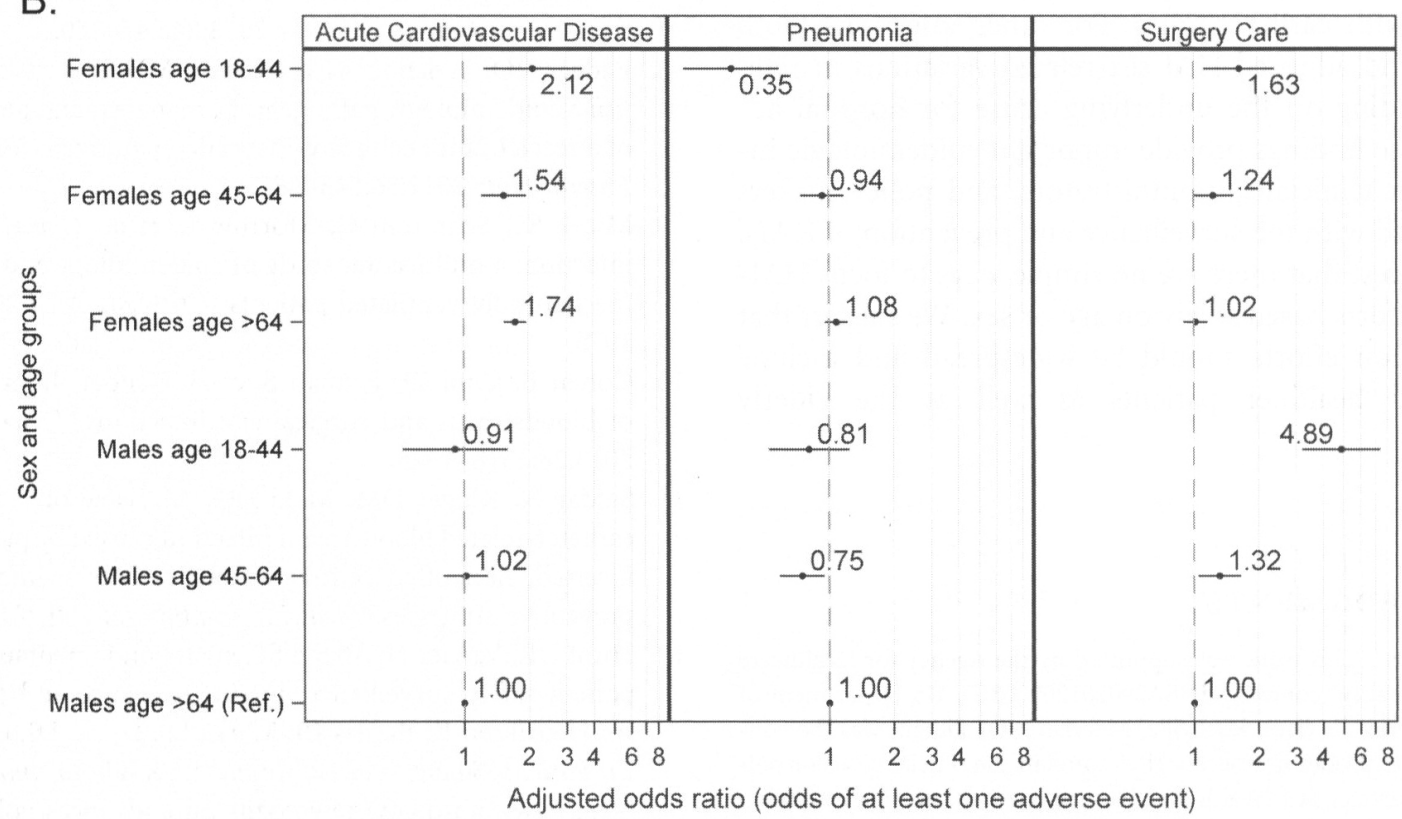

FIGURE 2. A, Adjusted odds ratio of rate of occurrence of healthcare-associated infections among all 6 age and sex groups. $B$, Adjusted odds ratio of patients having at least 1 healthcare-associated infection among all 6 age and sex groups. Ref, reference.

antibiotic use for pneumonia, antibiotic exposure might allow factors other than sex-related anatomic differences to assume a greater role (eg, catheter care and severity of illness). The finding of a markedly lower risk of HAI among younger females with pneumonia than among males is difficult to explain. We also found a much higher rate of HAI in young male surgical patients compared with all other groups. Young males are overrepresented among patients admitted to the hospital with major trauma, and there is evidence that trauma patients are at high risk of $\mathrm{HAI},{ }^{17}$ potentially contributing to this finding.

Our study has limitations that should be mentioned. Our methodology only detects HAIs that were diagnosed and documented, so some HAIs may not have been identified. Furthermore, there were low numbers of certain infections, limiting our ability to analyze sex- and age-related differences for most of the individual types of infections. We therefore used a composite measure of HAIs, which may have obscured 
sex and age related relationships that could exist in specific types of HAI. We also include only patients hospitalized with acute cardiovascular disease, pneumonia, or major surgery, so our results are not representative of all patients admitted to the hospital. Finally, our methodology does not allow us to differentiate between potentially preventable HAIs and those that were not preventable.

There are some notable strengths of this study. Our patient sample is composed of a randomly selected national sample. The results are based on chart abstraction by experienced abstractors, and HAI rates are calculated using rigorously tested algorithms developed by national clinical and patient safety experts. ${ }^{13}$ This methodology avoids the potential bias associated with other national HAI data sets, such as the NHSN, which relies on hospital reported data. ${ }^{19}$

In summary, we analyzed data from a large, randomly selected national sample of patients admitted to the hospital with acute cardiovascular disease, pneumonia, and major surgery to determine sex- and age-related differences in HAI rates. After adjusting for underlying comorbidities, we found no overall relationship between age and HAI rates. Unadjusted rates of CAUTI were higher among females than males, concordant with earlier studies. For other infections, both unadjusted and adjusted HAI sex-related variations in rates varied depending on the underlying cause for hospital admission. These findings provide important epidemiologic information for clinicians, administrators, and policy makers who are tasked with the surveillance and prevention of HAIs. The data suggest that there are no simple ways to focus HAIprevention efforts based solely on age or sex. We suggest that HAI prevention efforts should be widespread and include younger and healthier patients as well as the elderly population.

\section{ACKNOWLEDGMENTS}

Financial support. This work was supported by the Agency for Healthcare Research and Quality (contract HHSA290201200003C), US Department of Health and Human Services, Rockville, Maryland. Qualidigm was the contractor. The content does not necessarily represent the official views or policies of the Department of Health and Human Services, nor does mention of trade names, commercial products, or organizations imply endorsement by the US Government. The authors assume full responsibility for the accuracy and completeness of the ideas. The views expressed in the article represent the authors and not their respective institutional affiliations.

Potential conflicts of interest. M.L.M. reports having worked on various quality improvement and patient safety projects with Qualidigm, the Centers for Medicare and Medicaid Services, and the Agency for Healthcare Research and Quality, and his employer has received remuneration for this work. All other authors report no conflicts of interest relevant to this article. All authors submitted the ICMJE Form for Disclosure of Potential Conflicts of Interest, and the conflicts that the editors consider relevant to this article are disclosed here.

Address correspondence to Sheila Eckenrode, RN, Qualidigm, 1290 Silas Deane Highway, Suite 4A, Wethersfield, CT 06109 (seckenrode@qualidigm .org).

\section{REF E R E N C ES}

1. Centers for Disease Control and Prevention. Healthcare-associated infections: the burden. http://www.cdc.gov/hai/burden html. Accessed February 12, 2014.

2. Chenoweth $C$, Saint S. Preventing catheter-associated urinary tract infections in the intensive care unit. Crit Care Clin 2013; 29:19-32.

3. Pronovost P, Needham D, Berenholtz S, et al. An intervention to decrease catheter-related bloodstream infections in the ICU. N Engl J Med 2006;355:2725-2732.

4. Cook DJ, Walter SD, Cook RJ, et al. Incidence of and risk factors for ventilator-associated pneumonia in critically ill patients. Ann Intern Med 1998;129:433-440.

5. Siegel JD, Rhinehart E, Jackson M, et al. 2007 Guideline for isolation precautions: preventing transmission of infectious agents in health care settings. Am J Infect Control 2007;35:S65S164.

6. World Health Organization. Health care associated infections fact sheet world health 2014. http://www.who.int/gpsc /country_workgpsc_ccisc_fact_sheet_en.pdf. Accessed January 5, 2014.

7. Avci M, Ozgenc O, Coskuner S, et al. Hospital acquired infections (HAI) in the elderly: comparison with the younger patients. Arch Gerontol Geriatr 2012;54:247-250.

8. Badger VO, Ledeboer NA, Graham MB, et al. Clostridium difficile: epidemiology, pathogenesis, management, and prevention of a recalcitrant healthcare-associated pathogen. JPEN J Parenter Enteral Nutr 2012;36:645-662.

9. Micek ST, Schramm G, Morrow L, et al. Clostridium difficile infection: a multicenter study of epidemiology and outcomes in mechanically ventilated patients. Crit Care Med 2013;41:19681975.

10. Cohen B, Choi YJ, Hyman S, et al. Gender differences in risk of bloodstream and surgical site infections. $J$ Gen Intern Med 2013;28:1318-1325.

11. Safdar N, Kluger DM, Maki DG. A review of risk factors for catheter-related bloodstream infection caused by percutaneously inserted, noncuffed central venous catheters: implications for preventive strategies. Medicine (Baltimore) 2002;81:466-479.

12. Hunt DR, Verzier N, Abend SL, et al. Fundamentals of Medicare patient safety surveillance: intent, relevance and transparency. In: Henriksen K, Battles JB, Marks ES, Lewin DI, eds. Advances in Patient Safety: Vol. 2, From Research to Implementation. http://www.ahrq.gov/downloads/pub/advances/vol2/Hunt.pdf. Accessed March 30, 2012.

13. Wang $\mathrm{Y}$, Eldridge $\mathrm{N}$, Metersky $\mathrm{ML}$, et al. National trends in patient safety for four common conditions, 2005-2011. N Engl J Med 2014;370:341-351.

14. Gocmez C, Celik F, Tekin R, et al. Evaluation of risk factors affecting hospital-acquired infections in the neurosurgery intensive care unit. Int J Neurosci 2014;124:503-508.

15. Valles J, Peredo R, Burgueno MJ, et al. Efficacy of single-dose antibiotic against early-onset pneumonia in comatose patients who are ventilated. Chest 2013;143:1219-1225.

16. Niël-Weise B, van den Broek P. Antibiotic policies for shortterm catheter bladder drainage in adults. Cochrane Database Syst Rev 2005;20:CD005428.

17. Wallace WC, Cinat M, Gornick WB, et al. Nosocomial infections 
in the surgical intensive care unit: a difference between trauma and surgical patients. Am Surg 1999;65:987-990.

18. Dudeck MA, Weiner LM, Allen-Bridson $K$, et al. National
Healthcare Safety Network (NHSN) report, data summary for 2012, device-associated module. Am I Infect Control 2013;41: $1148-1166$. 